\title{
RELAÇÃO ENTRE ÍNDICE DE ÁREA FOLIAR E FRAÇÕES DE COMPONENTES PUROS DO MODELO LINEAR DE MISTURA ESPECTRAL, USANDO IMAGENS ETM+/LANDSAT ${ }^{1}$
}

\author{
ALEXANDRE C. XAVIER ${ }^{2}$, CARLOS A. VETTORAZZI ${ }^{3}$, RONALTON E. MACHADO
}

RESUMO: O índice de área foliar (IAF) é uma das mais importantes variáveis biofísicas da vegetação, estando relacionado diretamente com a evapotranspiração, com a produtividade da vegetação e com a interceptação da chuva pelo dossel. $\mathrm{O}$ objetivo deste trabalho foi analisar a relação do IAF de diversos tipos de cobertura do solo com Frações de Componentes Puros (FCPs) do Modelo Linear de Mistura Espectral (MLME). A área de estudo foi a microbacia hidrográfica do Ribeirão dos Marins, localizada no município de Piracicaba - SP. O IAF foi medido, no campo, com o equipamento LAI-2000, em 32 áreas com diferentes coberturas vegetais. A imagem utilizada foi do sensor ETM+ a bordo do satélite Landsat-7. No MLME, foram considerados três componentes puros (vegetação, solo e sombra), selecionados com o auxílio dos componentes principais. Como resultado, tem-se que o IAF variou de 0,47 a 4,48, quando consideradas todas as áreas. As relações do IAF com a fração do componente puro vegetação $\mathrm{F}_{\mathrm{VEG}}$ e com a fração do componente puro solo $\left(\mathrm{F}_{\mathrm{SOL}}\right)$ foram significativas, embora fracas. Ao considerar apenas dados de IAF de cana-de-açúcar, houve aumento da variação explicada tanto para $\mathrm{F}_{\mathrm{VEG}}$ como para $\mathrm{F}_{\mathrm{SOL}}$, sugerindo que a estratificação da vegetação pelo tipo pode melhorar a estimativa do IAF.

PALAVRAS-CHAVE: sensoriamento remoto, Modelo Linear de Mistura Espectral, LAI-2000.

\section{RELATIONSHIP BETWEEN LEAF AREA INDEX AND ENDMEMBER FRACTIONS FROM LINEAR SPECTRAL MIXTURE MODELLING, USING ETM+/LANDSAT IMAGES}

SUMMARY: The Leaf Area Index (LAI) is one of the most important biophysical variable of the vegetation for modeling, and it is directly related to evapotranspiration, vegetation yield and rain interception. The aim of this paper was to analyze the relationship between LAI and endmember fractions estimated by Linear Spectral Mixture Modelling (LSMM). The study area was a watershed, in Piracicaba, State of São Paulo, Brazil. LAI was measured with LAI-2000 equipment in 32 samples in the field with different vegetation cover. The LSMM was applied to a Landsat/ETM+ image, corrected for the atmospheric effects by 6S Model. Three endmembers were considered in the LSMM: vegetation, soil, and shade. The relationship between all LAIs and vegetation and soil fractions ( $\mathrm{F}_{\mathrm{VG}}$ and $\mathrm{F}_{\mathrm{SO}}$ ) were significant, although weak. The relationship between sugar-cane LAI with $\mathrm{F}_{\mathrm{VG}}$ and with $\mathrm{F}_{\text {SO }}$ showed better fits. These results indicated that the vegetation type had influenced on the LSMM and that the stratification by vegetation physiognomy is suggested to improve the LAI estimation. Relationship between LAI and shadow fraction was not statistically significant.

KEYWORDS: remote sensing, Linear Spectral Mixture Modelling, LAI-2000.

\footnotetext{
${ }^{1}$ Projeto e bolsa do primeiro autor financiados pela FAPESP, processos n⿳o 2000/09325-0 e no 98/144995, respectivamente.

${ }^{2}$ Engenheiro Agrícola, Pesquisador visitante, Instituto Nacional de Pesquisas Espaciais, Coordenação-Geral de Observação da Terra/Divisão de Sensoriamento Remoto, Av. dos Astronautas, 1758, São José dos Campos - SP, e-mail: xavier@ltid.inpe.br

${ }^{3}$ Engenheiro Agrônomo, Professor do Departamento de Engenharia Rural, ESALQ/USP, Piracicaba - SP.

${ }^{4}$ Engenheiro Agrícola, Departamento de Engenharia Rural, ESALQ/USP, Piracicaba - SP.

Recebido pelo Conselho Editorial em: 30-4-2002

Aprovado pelo Conselho Editorial em: 18-2-2004
} 


\section{INTRODUÇÃO}

A variável biofísica, índice de área foliar (IAF), definida como total de área foliar por área de superfície do terreno (WATSON, 1947), está diretamente relacionada com a evapotranspiração (ROSENBERG et al., 1983; LANG \& MCMURTRIE, 1992), sendo a principal variável de entrada descritora da vegetação (RUNNING \& COUGHLAN, 1988; TIKTAK \& GRINSVEN, 1995). O IAF também está diretamente relacionado com a capacidade de interceptação da chuva pelo dossel (KERGOAT, 1998; DIJK \& BRUIJNZEEL, 2001).

Técnicas de sensoriamento remoto têm-se mostrado ferramentas viáveis para estimar valores de IAF de maneira rápida e em escala regional, com nível aceitável de exatidão. Uma das formas mais simples de relacionar dados de sensoriamento remoto com IAF é por meio de índices de vegetação (TURNER et al., 1999). Um dos índices de vegetação amplamente utilizado é o NDVI ("Normalized Difference Vegetation Index"), porém esse índice é sensível às variações do substrato (HUETE, 1988). Frações de componentes puros (FCPs), oriundos de modelo linear de mistura espectral (MLME), mostraram ser menos sensíveis às variações de substrato que o NDVI para estimar o IAF (GARCÍAHARO et al., 1996), com uma dependência fraca ao ângulo zenital solar (HALL et al., 1995), podendo, dessa forma, ser um método mais eficaz de estimativa do IAF.

Quando fótons interagem com um simples componente dentro do campo de visada do sensor, a mistura pode ser modelada como a soma de cada componente. Porém, quando fótons são espalhados e interagem com múltiplos componentes, como ocorre com o espalhamento, pelas folhas, da radiação na região de comprimentos de onda do infravermelho próximo, a mistura tem um potencial de tornar-se não-linear (BOREL \& GERSTL, 1994). Nesse caso, modelos não-lineares podem ser utilizados (RAY \& MURRAY, 1996). Considerando o espalhamento múltiplo desprezível (ROBERTS et al., 1998), ou seja, os fótons interagem com cada um dos componentes individualmente dentro do campo de visada do sensor, pode-se aplicar o MLME, em que uma relação linear é usada para representar a mistura espectral de alvos dentro do campo de visada (pixel) do sensor remoto utilizado. Assim, para cada pixel, é possível obter uma decomposição espectral nas suas frações constituintes. Como uma imagem de sensoriamento remoto é uma matriz de pixels, é possível obter uma decomposição dessa imagem em subimagens que representam cada uma das frações espectrais constituintes dos pixels. Cada uma dessas frações denomina-se imagem fração de componente puro. As imagens fração de componentes puros considerados no MLME vêm sendo utilizadas para: identificar diferentes fases de plantios de eucalipto e diferentes espécies de pinheiro (HLAVKA \& SPANNER, 1995); correlacioná-las a variáveis biofísicas (HALL et al., 1995); identificar e mapear feições de degradação da terra relacionadas com processo de erosão do solo (METTERNICHT \& FERMONT, 1998); classificar imagens (CASALS-CARRASCO et al., 2000); mapear desflorestamento (SHIMABUKURO et al., 1994); estimar abundância de vegetação em área urbana (SMALL, 2001) e interpretar dados geológicos (ADAMS et al., 1986).

O objetivo deste trabalho foi avaliar a correlação entre valores de IAF de diferentes tipos de cobertura do solo existentes em uma microbacia rural com valores de frações de componentes puros oriundos de um MLME, gerados a partir de dados do sensor ETM+ do satélite Landsat 7.

\section{MATERIAL E MÉTODOS}

A área de estudo foi a microbacia hidrográfica do Ribeirão dos Marins, com aproximadamente $5.907 \mathrm{ha}$, situada entre as latitudes $22^{\circ} 41^{\prime} \mathrm{S}$ e $22^{\circ} 51^{\prime} \mathrm{S}$ e longitudes $47^{\circ} 40^{\prime} \mathrm{W}$ e $47^{\circ} 45^{\prime} \mathrm{W}$, no município de Piracicaba - SP. O clima regional é Cwa, subtropical úmido, conforme classificação de Köeppen, com verão chuvoso e inverno seco, e a precipitação média anual é de $1.278 \mathrm{~mm}$. As temperaturas médias mensais variam de $24,9{ }^{\circ} \mathrm{C}$, no verão, a $17,1{ }^{\circ} \mathrm{C}$ no inverno, com média anual de $21,4{ }^{\circ} \mathrm{C}$ (SENTELHAS et al., 1998). O relevo varia de ondulado a fortemente ondulado. 
O uso do solo é representado em grande parte por cana-de-açúcar e pastagens, apresentando também remanescentes de florestas, plantações de eucalipto (Eucalyptus spp.) e pequenas áreas com culturas anuais e horticultura. As áreas florestais são, em grande parte, remanescentes de floresta estacional semidecidual e floresta ripária (RODRIGUES, 1999). Na microbacia em estudo, a floresta limita-se quase que totalmente à floresta ripária, ocorrendo nas margens de cursos d'água, circundada ou pela cultura de cana-de-açúcar ou por pastagem. As espécies mais típicas presentes nas áreas de floresta ripária da região são figueiras (Ficus spp.), louveira (Cylolobium vecchii A. Samp.), guanandi (Colophyllum brasiliensis Camb.), ingá (Inga affinis DC. Hook et Arn), canela-do-brejo (Endlicheria paniculata (Spreng.) Macbr.), entre outras (RODRIGUES, 1999). As plantações de eucalipto totalizam uma pequena área, de aproximadamente 15 ha, localizadas principalmente na porção superior da bacia.

As áreas para avaliação do IAF foram selecionadas de acordo com os seguintes critérios: representar os diversos usos e coberturas do solo existentes na bacia; apresentar características homogêneas, como vigor, altura e distribuição das plantas; ter extensão compatível com a resolução espacial do sensor, e ser selecionado um número maior de amostras da cultura de cana-de-açúcar, pois essa apresenta a maior área de ocorrência e uma grande variabilidade no IAF para um mesmo período, devido à variabilidade de épocas de plantio.

Os valores de IAF em campo foram coletados com o equipamento LAI-2000 (LI-COR, 1992), entre os dias 15 e 20 de janeiro de 2001. Foram selecionados 32 pontos de coleta, dos quais: $22 \mathrm{em}$ cana-de-açúcar; três em eucaliptos; três em pastagem; dois em milho, e dois em floresta ripária.

\section{Processamento da imagem}

A imagem utilizada foi do sensor ETM+ a bordo do satélite Landsat-7 (órbita 220/ponto 76), gravada em 18 de janeiro de 2001, na mesma época da campanha de campo. Nela foi realizada a correção geométrica por meio de interpolador de primeiro grau de alocação de vizinho mais próximo. A projeção final do projeto foi UTM/SAD69. O erro final da correção foi de 0,5 pixel, ou seja, aproximadamente $12,5 \mathrm{~m}$. A reflectância de superfície foi estimada a partir do modelo 6S (VERMOTE et al., 1997). Os parâmetros de entrada no modelo 6S foram: modelo de atmosfera tropical, modelo continental de aerossóis e visibilidade horizontal de $15 \mathrm{~km}$. Para cada um dos 32 pontos onde se realizou a coleta de IAF em campo, foi adquirida a média de valores das FCPs de uma janela de $2 \times 2$ ou $3 \times 3$ pixels, de acordo com a forma e o tamanho de cada talhão estudado.

\section{Geração das frações dos componentes puros}

A resposta de cada pixel em qualquer comprimento de onda pode ser considerada como uma combinação linear das respostas de cada componente presente naquele. Desse modo, cada pixel contém informações sobre a fração e a resposta espectral de cada componente dentro da resolução unitária do terreno (SHIMABUKURO \& SMITH, 1991), podendo, dessa forma, aplicar-se o MLME, conforme eq.(1):

$$
r_{i}=\sum_{j=1}^{n}\left(a_{i j} x_{j}\right)+e_{i}
$$

em que,

$\mathrm{r}_{\mathrm{i}}$ - reflectância de superfície de um pixel, para a i-ésima banda espectral;

$\mathrm{a}_{\mathrm{ij}}$ - reflectância do j-ésimo componente puro do pixel para a i-ésima banda espectral;

$\mathrm{x}_{\mathrm{j}}$ - fração do j-ésimo componente puro no pixel;

$\mathrm{e}_{\mathrm{i}}$ - resíduo da modelagem para a $i$-ésima banda espectral;

$\mathrm{j}-1,2,3, \ldots, n$ (índice de componentes), e

i - $1,2,3, \ldots, m$ (índice de bandas espectrais consideradas). 
Cada um dos valores $r_{i}$ e $a_{i j}$ são admitidos como conhecidos. $O$ termo $x_{j}=0$ determina a ausência desse componente, e o valor $\mathrm{x}_{\mathrm{j}}=1$ indica que o pixel contém apenas o componente puro considerado. Para estimar a FCP $\left(\mathrm{x}_{\mathrm{j}}\right)$ do MLME, utilizam-se vários métodos, tais como: método dos mínimos quadrados com restrições e método dos mínimos quadrados ponderados (SHIMABUKURO, 1987), e componentes principais (ADAMS et al., 1986). Os resultados obtidos por essas técnicas são semelhantes (HOLBEN \& SHIMABUKURO, 1993). Neste trabalho, a estimativa das FCPs foi feita pela metodologia dos mínimos quadrados com restrição, baseada na minimização da função $\mathrm{F}=\sum_{i=1}^{m} e_{i}^{2}$, sendo que $m$ corresponde ao número de bandas espectrais do sensor ETM+. Maiores detalhes dessa solução podem ser encontrados em SHIMABUKURO \& SMITH (1991).

\section{Seleção dos componentes puros}

Neste trabalho, a seleção dos Componentes Puros (CPs) foi realizada com o auxílio do diagrama de dispersão entre os componentes principais (PC) (JOHNSON \& WICHERN, 1998) 1 e 2 (PC1 e PC2) gerados com as seis bandas de reflectância de superfície. Os dois primeiros PCs, PC1 e PC2, explicaram $97 \%$ da variação total da imagem. Os CPs foram selecionados nos vértices do diagrama de dispersão (Figura 1), e seus respectivos valores de reflectância (Figura 2) adquiridas em consulta às bandas individuais da imagem para o pixel selecionado. Dessa forma, obtiveram-se três CPs referentes à vegetação, ao solo e à sombra. Por meio da inspeção na imagem dos CPs selecionados e do padrão das suas respectivas curvas espectrais, observaram-se: a) o CP sombra correspondeu à área de água (rio Piracicaba), e o padrão da resposta espectral foi de baixos valores de reflectância, tanto na faixa espectral do visível como na do infravermelho; b) o CP solo foi localizado em área de solo exposto, sendo sua resposta espectral a maior na faixa do visível quando comparada aos demais CPs, e c) o CP vegetação correspondeu à área de cana-de-açúcar verde e sadia com baixos valores de reflectância de superfície na faixa do visível, devido à absorção da radiação pelos pigmentos nessa faixa e alto valor de reflectância na faixa do infravermelho próximo, devido ao espalhamento múltiplo da radiação pelas folhas do dossel.

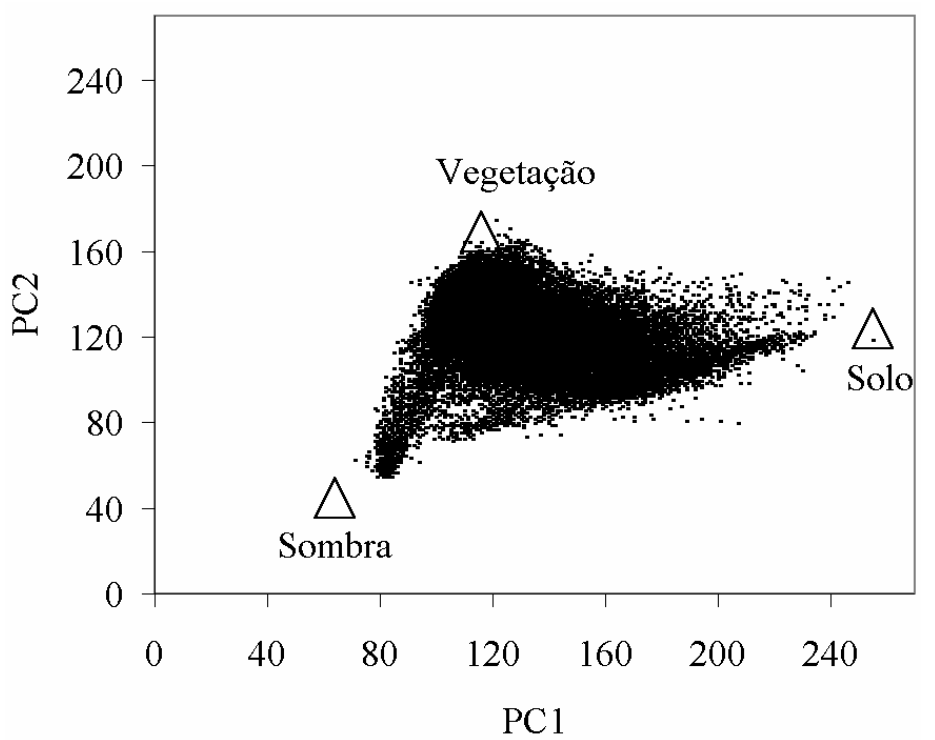

FIGURA 1. Diagrama de dispersão dos componentes principais 1 e 2 , sendo $\Delta$ os componentes puros localizados no vértice desse (64.000 pixels correspondente ao projeto). 


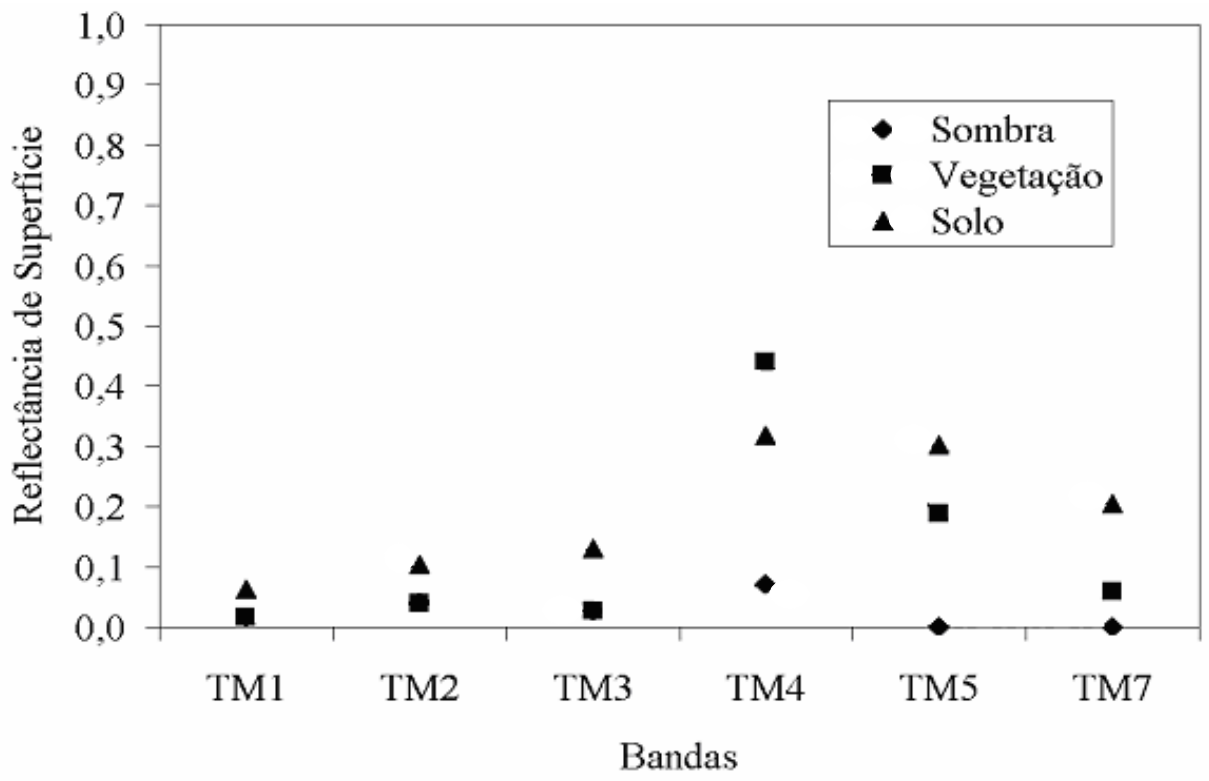

FIGURA 2. Diagrama de reflectâncias de superfície referente aos componentes puros vegetação, solo e sombra, selecionados a partir do diagrama dos componentes principais 1 e 2 .

\section{Avaliação estatística}

As relações entre o IAF e as FCPs (vegetação $\left(\mathrm{F}_{\mathrm{VEG}}\right)$, solo $\left(\mathrm{F}_{\mathrm{SOL}}\right)$ e sombra $\left(\mathrm{F}_{\mathrm{SOM}}\right)$ ) foram realizadas primeiramente de maneira individual, nas quais foram aplicados aos dados os modelos de regressão do tipo linear e potencial:

Modelo linear: $\mathrm{Y}=a \mathrm{IAF}+b$

Modelo potencial: $\mathrm{Y}=a \mathrm{IAF}^{b}$

em que,

Y - FCPs ( $\left.\mathrm{F}_{\mathrm{VEG}}, \mathrm{F}_{\mathrm{SOL}}, \mathrm{F}_{\mathrm{SOM}}\right)$, e

$a$ e $b$ - parâmetros dos modelos.

Esses modelos foram selecionados, pois já foram utilizados em análises do IAF com índices de vegetação e FCPs (TURNER et al., 1999; HALL et al., 1995). Os modelos polinomial quadrático e cúbico não foram utilizados, pois na análise preliminar dos dados não foi observada diminuição das FCPs com o aumento do IAF. Foi utilizada a análise de resíduo para identificar violação do modelo. Os modelos foram testados a $1 \%$ (valor $p$ ) (nível observado de significância) < 0,01) e escolhido aquele com maior coeficiente de determinação $\left(\mathrm{R}^{2}\right)$. Foi utilizada a análise de resíduos para identificar violação dos modelos (resíduo padronizado maior que três).

\section{RESULTADOS E DISCUSSÃO}

Os valores de IAF variaram de 0,47 a 4,48 para áreas de cana-de-açúcar e floresta ripária, respectivamente. Maiores detalhes sobre essas medidas podem ser encontrados em XAVIER \& VETTORAZZI (2003). Na Tabela 1, são apresentados os dados gerais de IAF para as diferentes coberturas do solo. 
TABELA 1. Valores de IAF com as respectivas frações de componentes puros derivados pelo método de mínimos quadrados com restrição: vegetação, solo e sombra.

\begin{tabular}{|c|c|c|c|c|}
\hline Uso do solo & LAI & $\mathrm{F}_{\text {VEG }}$ & $\mathrm{F}_{\mathrm{SOL}}$ & $\mathrm{F}_{\mathrm{SOM}}$ \\
\hline Cana-de-açúcar & 0,97 & 0,425 & 0,453 & 0,115 \\
\hline Cana-de-açúcar & 2,25 & 0,738 & 0,036 & 0,221 \\
\hline Cana-de-açúcar & 0,47 & 0,393 & 0,404 & 0,197 \\
\hline Cana-de-açúcar & 1,01 & 0,478 & 0,316 & 0,199 \\
\hline Cana-de-açúcar & 1,24 & 0,323 & 0,473 & 0,198 \\
\hline Cana-de-açúcar & 1,58 & 0,469 & 0,335 & 0,191 \\
\hline Cana-de-açúcar & 2,05 & 0,664 & 0,176 & 0,153 \\
\hline Cana-de-açúcar & 2,29 & 0,649 & 0,235 & 0,109 \\
\hline Cana-de-açúcar & 1,93 & 0,656 & 0,283 & 0,056 \\
\hline Cana-de-açúcar & 0,86 & 0,441 & 0,453 & 0,101 \\
\hline Cana-de-açúcar & 1,01 & 0,584 & 0,329 & 0,081 \\
\hline Cana-de-açúcar & 2,37 & 0,677 & 0,267 & 0,051 \\
\hline Cana-de-açúcar & 1,82 & 0,753 & 0,171 & 0,070 \\
\hline Cana-de-açúcar & 1,39 & 0,514 & 0,402 & 0,078 \\
\hline Cana-de-açúcar & 2,00 & 0,664 & 0,188 & 0,142 \\
\hline Cana-de-açúcar & 2,22 & 0,771 & 0,112 & 0,110 \\
\hline Cana-de-açúcar & 1,71 & 0,514 & 0,271 & 0,209 \\
\hline Cana-de-açúcar & 0,64 & 0,377 & 0,492 & 0,125 \\
\hline Cana-de-açúcar & 1,18 & 0,447 & 0,325 & 0,222 \\
\hline Cana-de-açúcar & 0,86 & 0,250 & 0,400 & 0,350 \\
\hline Cana-de-açúcar & 1,34 & 0,586 & 0,252 & 0,156 \\
\hline Cana-de-açúcar & 0,47 & 0,381 & 0,447 & 0,167 \\
\hline Pastagem & 1,49 & 0,454 & 0,491 & 0,049 \\
\hline Pastagem & 2,03 & 0,653 & 0,281 & 0,061 \\
\hline Pastagem & 1,09 & 0,637 & 0,192 & 0,166 \\
\hline Milho & 1,86 & 0,802 & 0,107 & 0,086 \\
\hline Milho & 1,26 & 0,389 & 0,560 & 0,045 \\
\hline Floresta Ripária & 3,21 & 0,712 & 0,143 & 0,139 \\
\hline Floresta Ripária & 4,48 & 0,709 & 0,092 & 0,192 \\
\hline Eucalipto & 2,78 & 0,660 & 0,032 & 0,307 \\
\hline Eucalipto & 1,99 & 0,557 & 0,234 & 0,203 \\
\hline Eucalipto & 3,11 & 0,554 & 0,050 & 0,390 \\
\hline
\end{tabular}

\section{Relações IAF-FCPs}

Ao analisar a imagem, o erro médio das imagens fração (SHIMABUKURO \& SMITH, 1995), estimado a partir dos CPs pelo método dos mínimos quadrados com restrição, utilizando os componentes puros, vegetação, solo e sombra, foi de $2 \%$. Na Figura 3, é apresentada a relação IAF$\mathrm{F}_{\mathrm{VEG}}$. Essa relação foi do tipo potencial com $\mathrm{R}^{2}=0,52$, sendo que, para maiores valores de IAF, houve aumento não-linear das $\mathrm{F}_{\mathrm{VEG}}$. $\mathrm{O}$ valor máximo das $\mathrm{F}_{\mathrm{VEG}}$ nas amostras estudadas foi de $80 \%$, em área de milho (IAF = 1,86). Considerando apenas os dados de IAF de espécies não-florestais (IAF $\leq 2,37$ ), a relação foi do tipo linear positiva (Tabela 2). Verificou-se um comportamento de linearidade para menores valores de IAF, que já fora observado por GARCÍA-HARO et al. (1996), que utilizaram

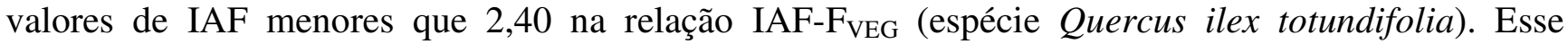
resultado pode demonstrar que o tipo de cobertura afeta o comportamento das frações desse 
componente puro, ou mais ainda, que para maiores valores de IAF haveria tendência de saturação da $\mathrm{F}_{\mathrm{VEG}}$, fazendo com que a relação também possa saturar a partir de determinado valor de IAF.

TABELA 2. Resumo dos resultados da análise de regressão simples da relação IAF com as frações dos componentes puros de vegetação $\left(\mathrm{F}_{\mathrm{VEG}}\right)$ e solo $\left(\mathrm{F}_{\mathrm{SOL}}\right)$.

\begin{tabular}{|c|c|c|c|c|c|c|}
\hline $\mathrm{Y}$ & IAF considerado & Modelo & $\mathrm{R}^{2}$ & $\begin{array}{c}\text { Erro- } \\
\text { Padrão }\end{array}$ & $\begin{array}{c}\text { Número de } \\
\text { Observações }\end{array}$ & valor $p$ \\
\hline \multirow{3}{*}{$\mathrm{F}_{\mathrm{VEG}}$} & Total $^{\mathrm{a}}$ & $0,4594 \mathrm{IAF}^{0,3860}$ & 0,52 & 0,20 & 32 & $<0,01$ \\
\hline & Não-florestais ${ }^{\mathrm{b}}$ & $0,2058 \mathrm{IAF}+0,2439$ & 0,62 & 0,09 & 27 & $<0,01$ \\
\hline & Cana-de-açúcar ${ }^{\mathrm{c}}$ & $0,2027 \mathrm{IAF}+0,2428$ & 0,70 & 0,08 & 22 & $<0,01$ \\
\hline \multirow{3}{*}{$\mathrm{F}_{\mathrm{SOL}}$} & Total & $-0,1269 \mathrm{IAF}+0,4992$ & 0,56 & 0,06 & 32 & $<0,01$ \\
\hline & Não-florestais & $-0,1652 \mathrm{IAF}+0,5540$ & 0,49 & 0,10 & 27 & $<0,01$ \\
\hline & Cana-de-açúcar & $-0,1667 \mathrm{IAF}+0,5500$ & 0,68 & 0,07 & 22 & $<0,01$ \\
\hline
\end{tabular}

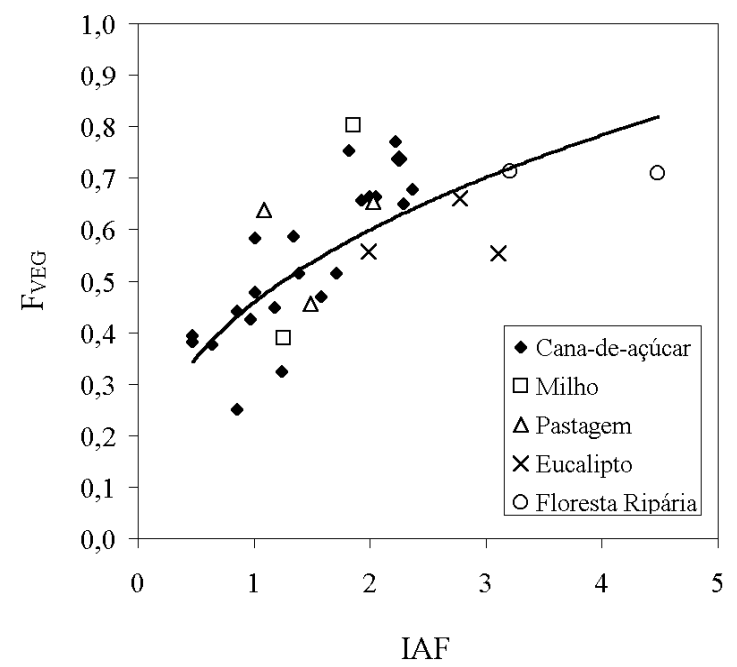

FIGURA 3. Diagrama de dispersão de todos os dados de IAF “versus" F FEG.

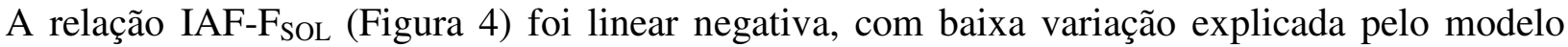
$\left(\mathrm{R}^{2}=0,56\right)$. $\mathrm{O}$ menor valor das $\mathrm{F}_{\mathrm{SOL}}$ para os dados analisados foi de $3 \%$, em plantação adulta de eucalipto (IAF $=2,78$ ). À medida que o IAF aumenta, diminui a área de solo exposto, acarretando diminuição da $\mathrm{F}_{\mathrm{SOL}}$. A estratificação dos dados de IAF para a cana-de-açúcar na relação com $\mathrm{F}_{\mathrm{SOL}}$ apresentou melhor ajuste (Tabela 2), com $\mathrm{R}^{2}$ de 0,68 e erro-padrão na estimativa do IAF, para a canade-açúcar, de $\approx 0,36 \mathrm{~m}^{2} \mathrm{~m}^{-2}$. O tipo de cobertura vegetal pode ter influenciado nas relações com o IAF, considerando $\mathrm{F}_{\mathrm{VEG}}$ e $\mathrm{F}_{\mathrm{SOL}}$. Isso sugere que a estratificação por tipo de cobertura pode melhorar o desempenho dessas relações.

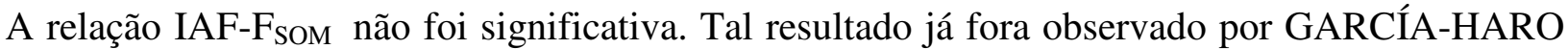

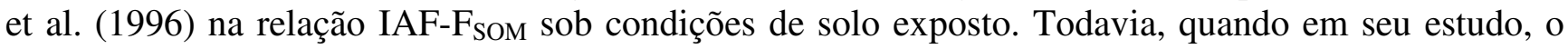
solo foi submetido a diferentes teores de carvão, passou a haver um aumento da $\mathrm{F}_{\mathrm{SOM}}$ até aproximadamente IAF de 1 e para maiores valores não foi observada diminuição significativa. Relação significativa entre IAF e F $_{\text {SOM }}$ já fora observado por HALL et al. (1995), que obtiveram a melhor relação do IAF de abeto-negro (Picea mariana) e choupo (Populus tremuloides), justamente com essa FCP. Os autores justificam que, em parte, esse resultado poderia ser explicado pela maior amplitude das $\mathrm{F}_{\mathrm{SOM}}$ em relação às demais frações. Percebe-se (Figura 5) que as plantações de eucalipto apresentam valores mais elevados de $\mathrm{F}_{\mathrm{SOM}}(0,30 \mathrm{em}$ média), o que as difere significativamente da 
média geral (0,15 em média). O fato de as plantações de eucalipto apresentarem maiores valores de F $_{\text {SOM }}$ pode ser explicado pela estrutura do seu dossel. Essas plantações encontram-se na fase adulta, como descrito anteriormente, sendo o espaçamento entre árvores de aproximadamente $3 \mathrm{~m}$, apresentando grandes aberturas no dossel, o que poderia aumentar a quantidade de sombras na área. As áreas de floresta ripária não apresentaram essa tendência de alta na $\mathrm{F}_{\mathrm{SOM}}$ no presente trabalho. Isso pode ser explicado pelo fato de esse tipo de cobertura não apresentar tantas aberturas como as plantações de eucalipto, embora seja uma cobertura que se apresenta na imagem com textura rugosa.

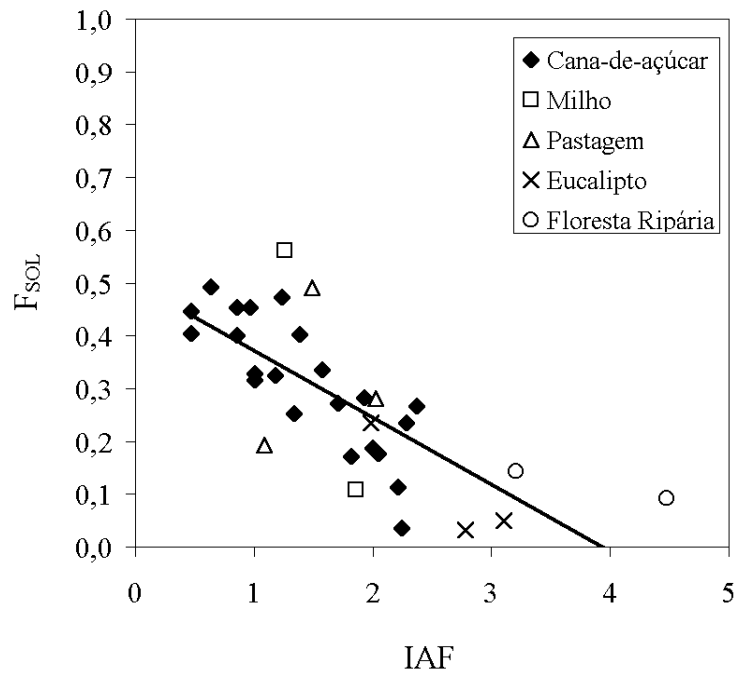

FIGURA 4. Diagrama de dispersão de todos os dados de IAF "versus" FSOL.

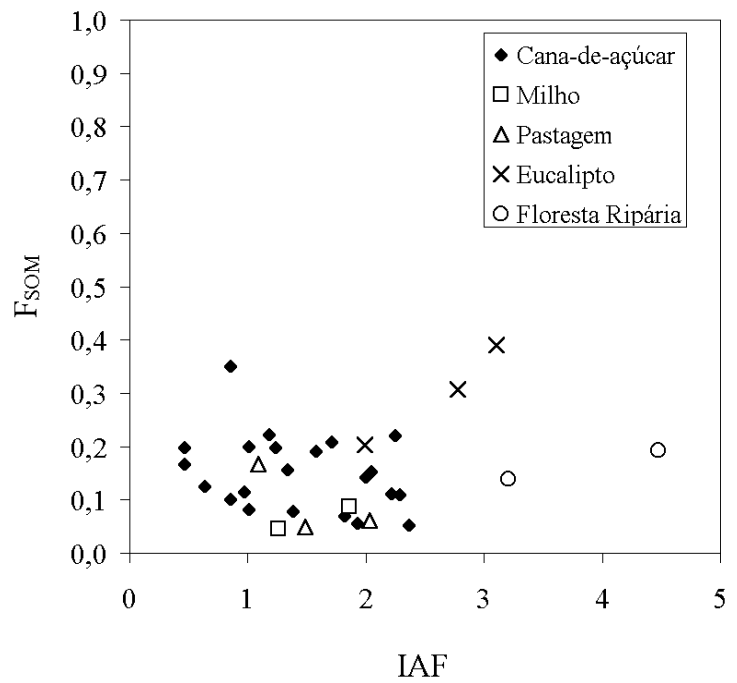

FIGURA 5. Diagrama de dispersão de IAF “versus" F FOM.

\section{CONCLUSÕES}

Houve relação significativa entre IAF de todos os tipos de vegetação encontrados na bacia com a $\mathrm{F}_{\mathrm{VEG}}$ e a $\mathrm{F}_{\mathrm{SOL}}$, embora fraca. A $\mathrm{F}_{\mathrm{SOM}}$ não apresentou relação significativa. Mostrou-se que a estratificação da vegetação pode melhorar a estimativa do IAF por F $_{\mathrm{VEG}}$ e $\mathrm{F}_{\mathrm{SOL}}$. 


\section{REFERÊNCIAS BIBLIOGRÁFICAS}

ADAMS, J.B.; SMITH, M.O.; JOHNSON, P.E. Spectral mixture modeling: a new analysis of rock and soil types at the Viking Lander 1 Site. Journal of Geophysical Research, Washington, v.91, p.8098-122, 1986.

BOREL, C.G.; GERSTL, S.A.W. Nonlinear spectral mixing models for vegetative and soil surfaces. Remote Sensing of Environment, New York, v.47, p.403-16, 1994.

CASALS-CARRASCO, P.; KUBO, S.; MADHAVAN, B.B. Application of spectral mixture analysis for terrain evaluation studies. International Journal of Remote Sensing, London, v.21, p.3039-55, 2000.

DIJK, A.I.J.M.; BRUIJNZEEL, L.A. Modelling rainfall interception by vegetation of variable density using an adapted analytical model. Part 2 - Model validation for a tropical upland mixed cropping system. Journal of Hydrology, Amsterdam, v.247, p.239-62, 2001.

GARCÍA-HARO, F.J.; GILABERT, M.A.; MELIA, J. Linear spectral mixture modeling to estimate vegetation amount from optical spectral data. International Journal of Remote Sensing, London, v.17, p.3373-3400, 1996.

HALL, F.G.; SHIMABUKURO, Y.E.; HUEMMRICH, K.F. Remote sensing of forest biophysical structure using mixture decomposition and geometric reflectance models. Ecological Applications, Washington, v.5, p.993-1013, 1995.

HLAVKA, C.A.; SPANNER, M.A. Unmixing AVHRR imagery to assess clear cuts and forest regrowth in Oregon. IEEE Transactions on Geosciences and Remote Sensing, New York, v.39, p.78895, 1995.

HOLBEN, B.N.; SHIMABUKURO, Y.E. Linear mixing model applied to AVHRR LAC data. In: SIMPÓSIO BRASILEIRO DE SENSORIAMENTO REMOTO, 7., 1993, Curitiba. Anais... São José dos Campos: Instituto Nacional de Pesquisas Espaciais, 1993. v.2, p.102-15.

HUETE, A.R. A soil-adjusted vegetation index (SAVI). Remote Sensing of Environment, New York, v.2, p.295-309, 1988.

JOHNSON, R.A.; WICHERN, D.W. Applied multivariate statistical analysis. 4. ed. New Jersey: Prentice Hall, 1998. 816 p.

KERGOAT, L. A model for hydrological equilibrium of leaf area index on a global scale. Journal of Hydrology, Amsterdam, v.212-13, p.268-86, 1998.

LANG, A.R.G; MCMURTRIE, R.E. Total leaf areas of single trees of Eucalyptus grandis estimated from transmittances of the sun's beam. Agricultural and Forest Meteorology, Amsterdam, v.58, p.7992, 1992.

LI-COR. LAI-2000 plant canopy analyzer: Instruction manual (Nebraska-Li-Cor). 1992. 166 p.

METTERNICHT, G.I.; FERMONT, A. Estimating erosion surface features by linear mixture modeling. Remote Sensing of Environment, New York, v.64, p.254-65, 1998.

RAY, T.W.; MURRAY, B.C. Nonlinear spectral mixing in desert vegetation. Remote Sensing of Environment, New York, v.55, p.59-64, 1996.

ROBERTS, D.A; GARDNER, M.; CHURCH, R.; USTIN, S.; SCHEER, G.; GREEN, R.O. Mapping Chaparral in the Santa Monica using multiple endmember spectral models. Remote Sensing of Environment, New York, v.65, p.267-79, 1998. 
RODRIGUES, R.R. A vegetação de Piracicaba e municípios do entorno. Piracicaba: IPEF, 1999. 18 p. (Circular Técnica, 189)

ROSENBERG, N.J.; BLAD, B.L.; VERMA, S.B. Microclimate: the biological environment. Washington: John Wiley, 1983. 459 p.

RUNNING, S.W.; COUGHLAN, J.C. A general model of forest ecosystem processes for regional applications. I - Hydrological balance, canopy gas exchange and primary production processes. Ecological Modelling, Amsterdam, v.42, p.125-54, 1988.

SENTELHAS, P.C.; MARIN, F.R.; PEREIRA, A.R.; ANGELOCCI, L.R.; VILLA NOVA, N.A.; BARBIERI, V. Análise dos dados climáticos e do balanço hídrico climatológico de Piracicaba. Piracicaba: DFM/ESALQ/USP, 1998. 81 p.

SHIMABUKURO, Y.E.; SMITH, J.A. The least-square mixing models to generate fraction images derived from remote sensing multispectral data. IEEE Transactions on Geosciences and Remote Sensing, New York, v.29, p.16-20, 1991.

SHIMABUKURO, Y.E.; HOLBEN, B.N.; TUCKER, C.J. Fraction images derived from NOAA AVHRR data for studying the deforestation in the Brazilian Amazon. International Journal of Remote Sensing, London, v.15, p.517-20, 1994.

SHIMABUKURO, Y.E. Shade images derived from linear mixing models of multispectral measurements of forested areas. 1987. 177 f. Dissertation (Ph.D.) - Colorado State University, Fort Collins, 1987.

SMALL, C. Estimation of urban vegetation abundance by spectral mixture analysis. International Journal of Remote Sensing, London, v.22, p.1305-34, 2001.

TIKTAK, A.; GRINSVEN, H.J.M. van. Review of sixteen forest-soil-atmosphere models. Ecological Modelling, Amsterdam, v.83, p.35-53, 1995.

TURNER, D.P.; COHEN, W.B.; KENNEDY, R.E.; FASSNACHT, K.S.; BRIGGS, J.M.

Relationships between leaf area index and Landsat TM Spectral Vegetation Indices across three temperate zone sites. Remote Sensing of Environment, New York, v.70, p.52-68, 1999.

VERMOTE, E.F.; TANRÉ, D.; DEUZÉ, J.L.; HERMAN, M.; MORCRETTE, J.J. Second simulation of the satellite signal in the solar spectrum, 6S: An overview. IEEE Transactions on Geosciences and Remote Sensing, New York, v.35, p.675-86, 1997.

WATSON, D.J. Comparative physiological studies on growth of field crops. I - Variation in net assimilation rate and leaf area between species and varieties, and within and between years. Annals of Botany, London, v.11, p.41-76, 1947.

XAVIER, A.C.; VETTORAZZI, C.A. Leaf area index of ground covers in a subtropical watershed. Scientia Agricola, Piracicaba, v.60, p.425-31, 2005. 